\title{
A COMPARISON OF THE TEMPERATURE AND EMISSION MEASURE OF X-RAY ACTIVE REGIONS WITH CORONAL MAGNETIC FIELDS
}

\author{
J. B. BURL and R. G. TESKE \\ Department of Astronomy, The University of Michigan, Ann Arbor, Mich. 48109, U.S.A. \\ and \\ E. B. MAYFIELD \\ Space Sciences Laboratory, The Aerospace Corporation, Los Angeles, Calif. 90009, U.S.A.
}

(Received 25 September, 1978; in revised form 16 January, 1979)

\begin{abstract}
The distribution of temperature and of emission measure in X-ray active regions relative to the coronal magnetic fields has been investigated. The position of maximum temperature and the position of maximum emission measure were found to lie along the magnetic neutral line, with the maximum temperature tending to lie above the position of an abrupt change in direction of the neutral line. Several simple structural models of these regions are compared to the emission measure. The total magnetic energy and the total emission measure appear to be related by a power law in the regions studied by us.
\end{abstract}

\section{Introduction}

For a long time it has been known that there is a close spatial relationship between $\mathrm{X}$-ray active regions in the corona and the associated magnetic fields. In general, the $\mathrm{X}$-ray emission is brightest near an active region's magnetic neutral line, where a bright core often connects the areas of opposite polarity (Krieger et al., 1971). Coronal magnetic fields calculated from the photospheric longitudinal components observed on magnetograms have been compared with X-ray images (Poletto et al., 1975; Krieger et al., 1976; Levine, 1976). It is found that there usually is a good correspondence of active region X-ray forms and inferred magnetic field structures, and that the X-rays are strongest where the field is strongest. Since the brightest $\mathrm{X}$-ray emission arises in those coronal volumes of greatest density and temperature, these studies imply that the magnetic field topology is closely related to the density and temperature structure. We expect this will be the case if the fields confine the plasma, with the highest gas pressures being exerted at places where the fields are the strongest.

It has been the purpose of this study to see if that view is correct. We have used $\mathrm{X}$-ray images from the MSFC/Aerospace S-056 X-ray telescope on ATM/Skylab (Underwood and McKenzie, 1977) to calculate the distributions of temperature and emission measure in coronal active centers, and have compared these with calculated magnetic field structures. The results strongly support the conclusions drawn from a direct comparison of $\mathrm{X}$-ray images and fields. 


\section{Data and Reductions}

Three small active regions were studied, McMath regions 12375,12378 , and 12381 , during the interval 5 to 10 June, 1973. All three regions had bipolar spot groups within them on at least one day but were otherwise not magnetically complex, and all underwent considerable evolution during the period of observation. Only 12375 produced confirmed flares, and all three of these were subflares. We believe our conclusions will be valid for the entire lifetime of such small, quiescent active centers.

Contour diagrams of temperature and emission measure were prepared for the regions as described by Teske and Mayfield (1976). Davies et al. (1975) and Pye et al. (1979) demonstrated that one may derive from X-ray imagery an equivalent temperature, averaged along a line of sight, that agrees with a spectroscopically derived temperature within $10 \%$. With that equivalent temperature one can get an equivalent emission measure, which is the emission measure needed if all the material emits at the equivalent temperature. Pye et al. (1979) found that the equivalent emission measure derived from S-054 X-ray images was however only in rough accord with a spectroscopically derived emission measure. Our conclusions here will be based upon relative emission measures and temperatures, so that absolute errors are not a consideration. Effective pixel sizes in these data are $2.8^{\prime \prime} \times 2.8^{\prime \prime}$ arc.

Chiu and Hilton's (1977) procedure was used to calculate coronal magnetic fields from KPNO magnetograms under the assumption that the currents in the coronal are small and contribute only negligibly to those fields. The validity of the potential approximation has been discussed by Poletto et al. (1975). Except in a small number of cases (Levine, 1976) it has been found that potential fields are useful in characterizing the forms of coronal X-ray structures (Krieger et al., 1976). We prepared contour diagrams of $|\mathbf{B}|$ at altitudes of 10000,20000 , and $30000 \mathrm{~km}$ above the photosphere, with an effective pixel size of $7^{\prime \prime} \times 7^{\prime \prime}$ arc, for comparison with the X-ray data. Relative positions of structures are thus known to approximately this dimension.

\section{Results: Emission Measure and Temperature in the Magnetic Fields}

\subsection{Static PROperties}

In the potential fields mapped by us, the forms of isogauss contours look qualitatively similar at the different altitudes; this is especially true of the location and shape of the magnetic neutral line. Hence we focussed our attention on the magnetic maps of $10000 \mathrm{~km}$ altitude.

The positions of maximum temperature $T_{\max }$ and maximum emission measure per pixel $\left(N_{e}^{2} \Delta V\right)_{\max }$ lay very close to the neutral line of these regions, to within $8000 \mathrm{~km}$ (11" arc). Figure 1a and b show superposed data for 6 June and 9 June, 1973. Although the locations of $T_{\max }$ and $\left(N_{e}^{2} \Delta V\right)_{\max }$ do not always occur at the same position on the $\mathrm{X}$-ray maps, any displacement between the two positions is along the neutral line. 

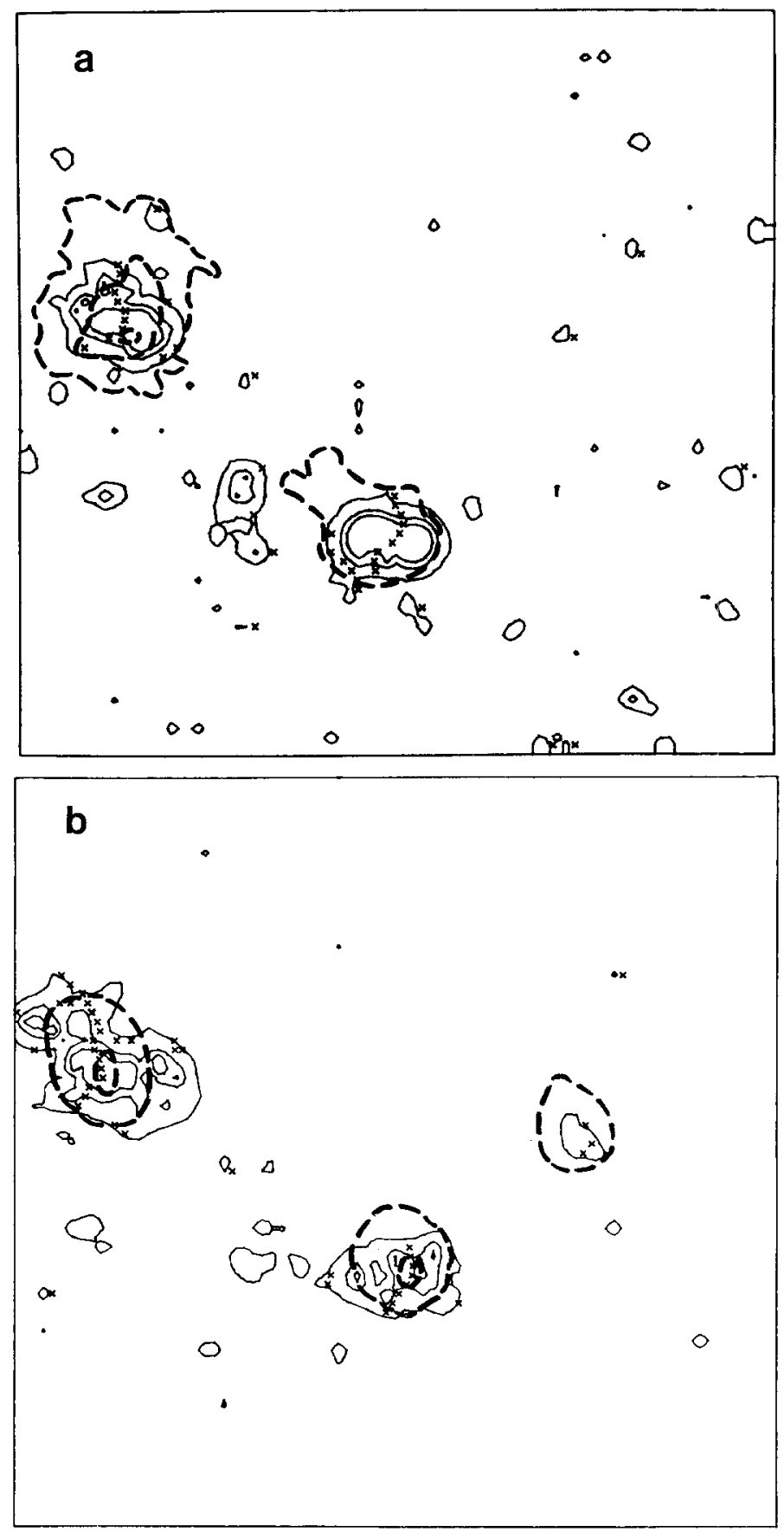

Fig. 1. (a) 6 June 1973 and (b) 9 June 1973. The solid curves are isogauss contours of $|\mathbf{B}|$ at 20,50 , and $80 \mathrm{G}$, as calculated for $10000 \mathrm{~km}$ altitude from KPNO magnetograms taken near 19:00 UT. Positions at which $B_{z}=0$ are indicated by an ' $\times$ ' to locate the magnetic neutral line. Isocontours of temperature at $T_{6}=2.5,3.0$, and 3.5 are dashed. They were calculated from X-ray images secured at (a) 14:23 UT on 6 June and (b) 13:47 UT on 9 June. North is up and west is to the right. The active regions are: 12375, upper left; 12378 in the center; 12381 on the right. 
During about half the days, the neutral line in 12375 and 12378 made an abrupt change of direction of between $30^{\circ}$ and $60^{\circ}$ (Figure 1), and the position of $T_{\max }$ was found to be at the vertex of the angle, to within about $5000 \mathrm{~km}$. McIntosh et al. (1976) have stated that the brightest X-ray loops in an active region occurred over that portion of the neutral line 'with a convolution'.

It is possible that the location of an abrupt change in neutral line direction, as calculated in the current-free case, is an indication that the real fields locally depart towards a non-potential configuration. The currents which then must flow could be a source of local heating in the corona, accounting for our results but not those of McIntosh et al. (1976).

All of the contours of temperature and emission measure extend further along the direction of the neutral line than perpendicular to it - the contours are rough ellipses with their major axes along the neutral line (Figure 1). Since the emission measure contours are decidedly more elliptical than the temperature contours, we infer that emission measure depends upon position relative to the neutral line more than does temperature.

\subsection{DyNAMIC PROPERTIES}

Region 12375 experienced a rotation relative to the photospheric surface of about $30^{\circ}$ between 6 June and 9 June (Figure 1). Projection effects were responsible for an apparent rotation of some $6^{\circ}$ of this; the influence of differential solar rotation was quite minor. The magnetic neutral line and the major axes of the elliptical contours of emission measure and temperature all rotated by the same amount, in the sense that material at the region's low-latitude edge moved westwards faster than did material the region's northern edge. This shows rather remarkably that a rotation of the fundamental field structure carries the coronal X-ray plasma loops right along with itself.

Our computed isogauss contours for region 12375 show islands of strong field in the $p$ and $f$ polarities (Figure 1b) which we have interpreted as indicating the location of the feet of a major magnetic flux tube. X-ray images taken within six hours of the magnetogram of Figure $1 \mathrm{~b}$ do not show an obvious corresponding $\mathrm{X}$-ray feature, nor are there corresponding temperature or emission measure features. During the interval 7 June to 9 June these magnetic islands spread apart at the rate of some $1.7 \times 10^{4} \mathrm{~km} \mathrm{day}^{-1}$. McIntosh et al. (1976) have estimated the rate of spreading of the feet of X-ray loops by a direct analysis of the images. If we interpret the rate of spreading of the magnetic islands in our data as the spreading of feet of a magnetic arch, we find the rate is less than a factor of two faster than the rate of spreading of $\mathrm{X}$-ray loop foot-points in one week old regions as measured by McIntosh et al. (1976). Thus the evidence that this is a major magnetic arch without an X-ray counterpart is circumstantial but not compelling.

Changes in the extent of the emission measure contours of these active regions proceed preferentially along the magnetic neutral line. During the six days of this study region 12375 goes through a fading and resurgence while 12378 and 12381 are 
born and die. The emission measure contours maintain an approximately fixed size in a direction perpendicular to the neutral line during all stages of evolution. In contrast, there is considerable change in the extent of the emission measure contours along the direction of the neutral line. This is explained if the regions are composed of a series of loops with their foot-points located in approximately the same position and with their tops spreading along the neutral line (see Figure 6 in Levine (1976) and Figure 6 and 10 in Poletto et al. (1975)). The loops which extend farthest along the neutral line would lie almost parallel to the solar photosphere. Our data do not indicate whether evolutionary elongation of the emission measure contours is caused by a filling of successively flatter magnetic flux tubes or if the same loops remain filled and X-ray bright, their angle to the photosphere decreasing progressively with time. Levine (1976) too has discussed this question but reached no conclusion. As the regions decay, the emission measure contours become less elliptical once again, indicating either an emptying of the outer loops or an increase in the angle they make with the solar surface.

Levine (1976) suggests that field configurations in which flux loops lie at small angles to the photosphere are systems which depart considerably from the potential case. On the other hand the current-free calculations of Poletto et al. (1975) show just such loops, although they are small ones. Our data do not permit an estimation of the amount of departure of these regions from the idealized potential configuration.

\subsection{EVOLUTION OF MAGNETIC ENERGY CONTENT AND EMISSION MEASURE}

The potential configuration we have calculated by Chiu and Hilton's (1977) method represents the lowest energy state of a magnetic system having the observed photospheric longitudinal components. A procedure due to Schmidt (1964) may be

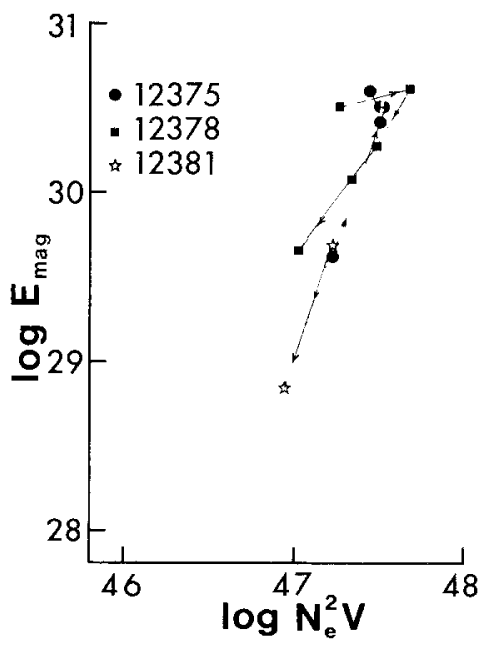

Fig. 2. Evolution of magnetic energy and equivalent emission measure in the three active regions. Arrows indicate day-to-day changes. Only two days of data were usable for McMath region 12381. 
used to obtain by straightforward integration the total magnetic energy stored in that system. There is no clear justification however for assuming the energy thus estimated to be in fact correct; nonpotential fields will store additional energy while still maintaining the same photospheric longitudinal components. We have indicated two reasons for thinking that the fields involved with the regions being studied here may depart from a purely potential system. These coronal active regions are however small, simple and quiescent, and such departures may be minor. So it may be appropriate, as a first approximation, to estimate the total magnetic energy as if it were stored in a current-free field.

We have done so using Schmidt's (1964) method, and Figure 2 compares the resulting energies with the total emission measures for the evolving active centers. $E_{\text {mag }}$ represents the energy of the entire system above the photosphere. In so far as these data may portray the physical conditions correctly, we note that the active regions have a rough tendency to evolve along tracks on which emission measure and total magnetic energy are related by a power law.

\section{Comparison of Emission Measures with Simple Geometric Loop Models}

Three approximations to the structure of X-ray loops have been used in the literature. In one case the loops are considered to be of constant thickness (e.g. Landini and Monsignori-Fossi, 1975; Rosner et al., 1978). A second kind of model envisions that a vertical cross-section occupies the space between two truncated conics, such as parabolas, with identical foot points (e.g. Parkinson, 1972). In a vertical cross-section of the third kind of model, the plasma occupies the space between lines of force of a magnetic dipole (e.g. Antiochos and Sturrock, 1976).

We have attempted to use our emission measure distributions to discern which of these conceptual models might be useful. Three assumptions were made. We assumed that the active regions consist of individual loops which are not resolved in the emission measure maps, and that these loops are parallel to the loops that can be seen crossing the neutral line in the imagery, an apparently reasonable assumption. Secondly we assumed that the equivalent emission measures which we used are not so badly in error as to invalidate tests of the models. We cannot check this assumption, and cannot discount the possibility it is wrong. Lastly we assume that electron density is constant along the length of a loop. This is reasonable since in these small active regions the loop dimensions are smaller than a coronal scale height. With this last assumption the models place constraints on how emission measure in the pixels $\left(N_{e}^{2} \Delta V \sim N_{e}^{2} \Delta z\right)$ can vary along the projected loops. By taking ratios of emission measures along the same loop the electron density is eliminated, leaving a quantity that depends only upon the vertical cross-section of the loop. No complications occur if the loops are nested, so long as the same model applies to all.

We used data from 7 and 8 June, dates on which the line of sight to Earth made an angle with the local solar radius of $12^{\circ}$ or less in each active region. Thus, provided 
that the loop thicknesses are small relative to their lengths, projection effects will be minor.

At the outset the loop model of constant thickness can be eliminated. It requires that the emission measure along a line of sight is a minimum near the neutral line, at the top of the loop, and that it increase outwards away from the neutral line, a situation which is in sharp contrast to observation.

Our emission measure data could not be made to satisfy the second model. Specifically, vertical cross-sections that were cut out by segments of parabolas and by segments of ellipses were tested. These are one-parameter families for which a unique result should be obtained at all positions along the loop. There was no unique parameter that could be found for any loop, however.

On the other hand the data could be made to fit loops that were segments of a dipole field, or at least dipole-like. Two parameters are needed to achieve a fit, but ratios of emission measure yield only one. However, the trend of this parameter away from the neutral line - and loop tops - very closely follows that predicted for a dipole field. The data show slightly different trends on each side of the neutral lines, indicating that the structure of the regions is more complex than is suggested by a simple dipole field model, but this does not undermine its conceptual usefulness.

\section{Summary}

Using X-ray images of the Sun we prepared two-dimensional maps of emission measure and temperature in three coronal active regions chosen for study because they were small, simple and free from flare activity. These maps have been compared with maps of coronal magnetic field structure calculated in the potential approximation. Our data span an interval of six days in June, 1973. $T_{\max }$ and $\left(N_{e}^{2} \Delta V\right)_{\max }$ were found to lie above the neutral line of the magnetic field, with the maximum temperature preferentially lying above the position of an abrupt change in the direction of the neutral line.

Growth and decay of the regions is accompanied by changes in the emission measure contours which are more pronounced when their extent is measured along the neutral line than when it is measured perpendicular to the neutral line. We have supposed this is caused by unresolved plasma loops whose foot points lie on opposite sides of the neutral line and which are oriented nearly parallel with the photospheric surface. It is possible these nearly horizontal plasma tubes are successively filled as the region grows, and emptied as it decays. Our data cannot distinguish between this possibility and the possibility that we are seeing the same plasma tubes leaning at successively different angles with the photosphere as the regions grow and decline.

Although these observations may indicate that the embedded magnetic fields depart to some extent from a current-free configuration, we have calculated the total magnetic energy in these coronal volumes under the assumption that such departures are minor. We find that the total magnetic energy tends to be related to the total equivalent emission measure by a power law. 
As a means of testing possible geometric models for X-ray loops we examined the emission measure distributions in directions away from the magnetic neutral line and parallel to loops visible in the X-ray images. The data were inconsistent with a geometric model with constant loop cross-section and with a model whose vertical cross-section is defined by intersecting conics. A model in which the loops are segments outlined by magnetic dipole fields was found to be qualitatively consistent with the data, but a more detailed study will be needed to verify this conclusion.

\section{Acknowledgements}

The authors especially thank Miss Joanna Frawley for extensive help in computer programming and plotting. This work was performed under NASA contract NAS829602.

\section{References}

Antiochos, S. K. and Sturrock, P. A.: 1976, Solar Phys. 49, 359.

Chiu, Y. T. and Hilton, H. H.: 1977, Astrophys. J. $212,873$.

Davis, J. M., Gerassimenko, M., Krieger, A. S., and Vaiana, G. S.: 1975, Solar Phys. $45,394$.

Krieger, A. S., Vaiana, G. S., and van Speybroeck, L. P.: 1971, in R. Howard (ed.), 'Solar Magnetic Fields', IAU Symp. 43, 397.

Krieger, A. S., de Feiter, L. D., and Vaiana, G. S.: 1976, Solar Phys. 47, 117.

Landini, M. and Monsignori-Fossi, B. C.: 1975, Astron. Astrophys. 42, 213.

Levine, R. H.: 1976, Solar Phys.: 46, 159.

McIntosh, P. S., Krieger, A. S., Nolte, J. T., and Vaiana, G. S.: 1976, Solar Phys. 49, 57.

Parkinson, J. H.: 1972, Solar Phys. 28, 487.

Poletto, G., Vaiana, G. S., Zombeck, M. V., Krieger, A. S., and Timothy, A. F.: 1975, Solar Phys. 44, 83. Pye, J. P., Evans, K. D., Hutcheon, R. J., Gerassimenko, M., Davis, J. M., Krieger, A. S., and Vesecky, J. F.: 1979, submitted to Solar Phys.

Rosner, R., Tucker, W. H., and Vaiana, G. S.: 1978, Astrophys. J. 220, 643.

Schmidt, H. U.: 1964, in W. N. Hess (ed.), AAS-NASA Symposium on the Physics of Solar Flares, p. 107.

Teske, R. G. and Mayfield, E. B.: 1976, Astrophys. J. 210, L153.

Underwood, J. H. and McKenzie, D. L.: 1977, Solar Phys. 53, 417. 